\title{
Structural and microstructural characteristics of B-doped PbTe semiconductor
}

\author{
Jovica N. Stojanovic \\ Institute for Technology of Nuclear and Other Raw Mineral Materials, Applied \\ Mineralogy Unit, Franche d'Eperey 86, P.O. Box 390, 11000 Belgrade, Serbia \\ E-mail: j.stojanovic@itnms.ac.yu, Phone: +381-11-3691-722
}

\begin{abstract}
The main task of this paper was accurate determination of structural and microstructural parameters of B-doped PbTe semiconductor (" $p$ " type). Four samples (undoped PbTe, and three doped with initial B contents: $1 \%, 3 \%$ and $8 \%$ ) were synthesized using the Bridgman method and analysed using X-ray powder diffraction (XRD) technique. Structural features were obtained using the Rietveld method and microstructural by diffraction-line broadening methods. Microstructural measurements contain both crystallite domain sizes and microstrain calculations obtained by the Warren-Averbach and the simplified integral-breadth methods.
\end{abstract}

Keywords: B-doped PbTe semiconductor, the Rietveld method, microstructural measurements.

\section{INTRODUCTION}

$\mathrm{PbTe}$ is a narrow-gap semiconductor. The research in doping of semiconductor materials and hereby modified properties is one of the most significant tasks of modern semiconductor physics. Great consideration is given to optical, photoelectric and magnetic features of $\mathrm{A}^{\mathrm{IV}} \mathrm{B}^{\mathrm{VI}}$ compounds doped with some elements from the III group (B, Al, Ga, In, Tl). The most studied compounds, thus far, are In, Ga and Tldoped $\mathrm{A}^{\mathrm{IV}} \mathrm{B}^{\mathrm{VI}}$ compounds. B-doped PbTe is one serial semiconductor material, important in manufacturing of laser and detector infrared (IR) optoelectronics within wavelength from 1 to $40 \mu \mathrm{m}$. 
The scope of the paper is to give an answer with the reference to the issues of how and where $\mathrm{B}$ atoms have sited into $\mathrm{PbTe}$ structure. Rietveld refinement method was used for structural and crystallite domain-size as well as crystal lattice microstrain calculations for microstructural determinations. Both crystallite domain size and microstrain measurements were calculated towards obtaining the crystallographic direction distributions of B atoms.

The Warren-Averbach method and corresponding microstrain calculations were applied in diffraction-line broadening measurements for surface-weighted $\left(\langle D\rangle_{S}\right)$ and volume-weighted $\left\langle D>_{V}\right.$ ), crystallite domain sizes. The referred measurements were also done by applying "simplified" integral-breadth methods: 1) broadenings originated due to both crystallite domain sizes and microstrains, which correspond to the Cauchy function, 2) the Gauss function and 3) both broadenings originated due to crystallite domain sizes that correspond to the Cauchy function and microstrains that correspond to the Gauss function. The last one provides for an excellent theoretical basis and it is widely applied in various Rietveld analysis softwares intended to microstructural calculations.

\section{EXPERIMENT AND ANALYTICAL METHODS}

B-doped PbTe samples were synthesized using the Bridgman method. $\mathrm{Pb}$ and $\mathrm{Te}$ were applied in an ingot form, of a normal $99.99 \mathrm{wt} \%$ purity. So derived samples were further powdered for XRD analyses. Four samples were synthesized in the Institute of Technical Sciences of the Serbian Academy of Sciences and Arts: undoped $\mathrm{PbTe}$, PbTe doped with $\mathrm{B}$ (1 at.\%, 3 at.\% and 8 at.\%). X-ray intensity measurements were carried out on a PHILIPS PW 1710 automated diffractometer. The powder diffraction data are given in Table 1. The FULLPROF software [2] was used for the Rietveld structure refinement and BREADTH [1] for the diffraction-line broadening measurements.

\section{RESULTS AND DISCUSSION}

\section{Rietveld structure refinement}

PbTe is cubic, space group $F m \overline{3} m$ with $\mathrm{Z}=4$. Both $\mathrm{Pb}$ and $\mathrm{Te}$ atoms are in fixed positions at $1 / 2 \frac{1}{2} \frac{1}{2}$ and 000 respectively, and both atoms are in octahedral 
coordination.

Table 1. Rietveld powder diffraction data for B-doped $\mathrm{PbTe}$.

\begin{tabular}{|c|c|c|}
\hline \multicolumn{3}{|l|}{ Sample characterization } \\
\hline Name (chemical, mineral) & \multicolumn{2}{|l|}{ Lead telluride (altaite) } \\
\hline Empirical formula & \multicolumn{2}{|l|}{$\mathrm{PbTe}$} \\
\hline Source/preparation & \multicolumn{2}{|l|}{ synthetic } \\
\hline \multicolumn{3}{|l|}{ Technique } \\
\hline $\begin{array}{l}\text { Radiation type, source } \\
\text { Monochromator }\end{array}$ & \multicolumn{2}{|c|}{$\begin{array}{l}\text { X-rays, } \mathrm{Cu} \lambda \text { value used } 1.54060 \AA \mathrm{K} \alpha_{1} / 1.54439 \mathrm{~K} \alpha_{2} \\
\text { diffracted beam } \\
\text { graphite } \\
\text { monochromator }\end{array}$} \\
\hline Detector (film, scint. etc) & \multirow{2}{*}{\multicolumn{2}{|c|}{$\begin{array}{l}\text { proportional } \\
\text { vertical diffractometer } \\
\text { divergence slit } 1^{\circ} \\
\text { receiving slit } 0.1 \mathrm{~mm} \\
\text { soller slit } 1^{\circ}\end{array}$}} \\
\hline $\begin{array}{l}\text { Instrument description } \\
\text { (type, slits) }\end{array}$ & & \\
\hline $\begin{array}{l}\text { Instrumental profile } \\
\text { breadth }\end{array}$ & $0.10^{\circ} 2 \theta$ & $\begin{array}{l}25 \\
\pm 1\end{array}$ \\
\hline $\begin{array}{l}\text { Specimen form } / \text { particle } \\
\text { size } \\
\text { Range of } 2 \theta \\
\text { Specimen motion }\end{array}$ & $\begin{array}{l}\text { edge loaded powder } /<10 \mu \mathrm{m} \text { particle size } \\
\text { from } 20^{\circ} \text { to } 140^{\circ} 2 \theta, 4 \mathrm{~s} \text { per step } \\
\text { none }\end{array}$ & \\
\hline
\end{tabular}

There were no other phases except PbTe during the treatments. Even the reflection displacements did not occur. Therefore, the conclusion is that B atoms haven't deranged the PbTe structure when sited in it. (200) reflection showed strong, while (220) and (222) reflection showed appreciably weaker preferred orientation effects in all sample. The observed (circles), calculated (continuous line) and difference powder diffraction profiles for all analysed samples are shown in Figure 1. The Rietveld agreement factors are presented in Table 2.

Table 2. Rietveld refinement agreement factors

\begin{tabular}{lcccc}
\hline & $\mathrm{PbTe}$ & $\mathrm{PbTe}+\mathrm{B}(1 \%)$ & $\mathrm{PbTe}+\mathrm{B}(3 \%)$ & $\mathrm{PbTe}+\mathrm{B}(8 \%)$ \\
\hline$R_{\exp }$ & 11.4 & 10.7 & 11.3 & 10.5 \\
$R_{p}$ & 13.3 & 12.7 & 14.2 & 12.4 \\
$R_{w p}$ & 14.3 & 14.1 & 14.9 & 14.8 \\
$R_{B}$ & 6.12 & 5.58 & 5.69 & 5.82 \\
$R_{F}$ & 6.47 & 5.55 & 5.28 & 6.48 \\
$G o_{f} F$ & 1.6 & 1.7 & 1.6 & 1.7 \\
\hline
\end{tabular}

The unit-cell parameter of pure $\mathrm{PbTe}$ is in a good agreement with all B-doped 
samples and literature data [4] as well. The isotropic displacement parameters of

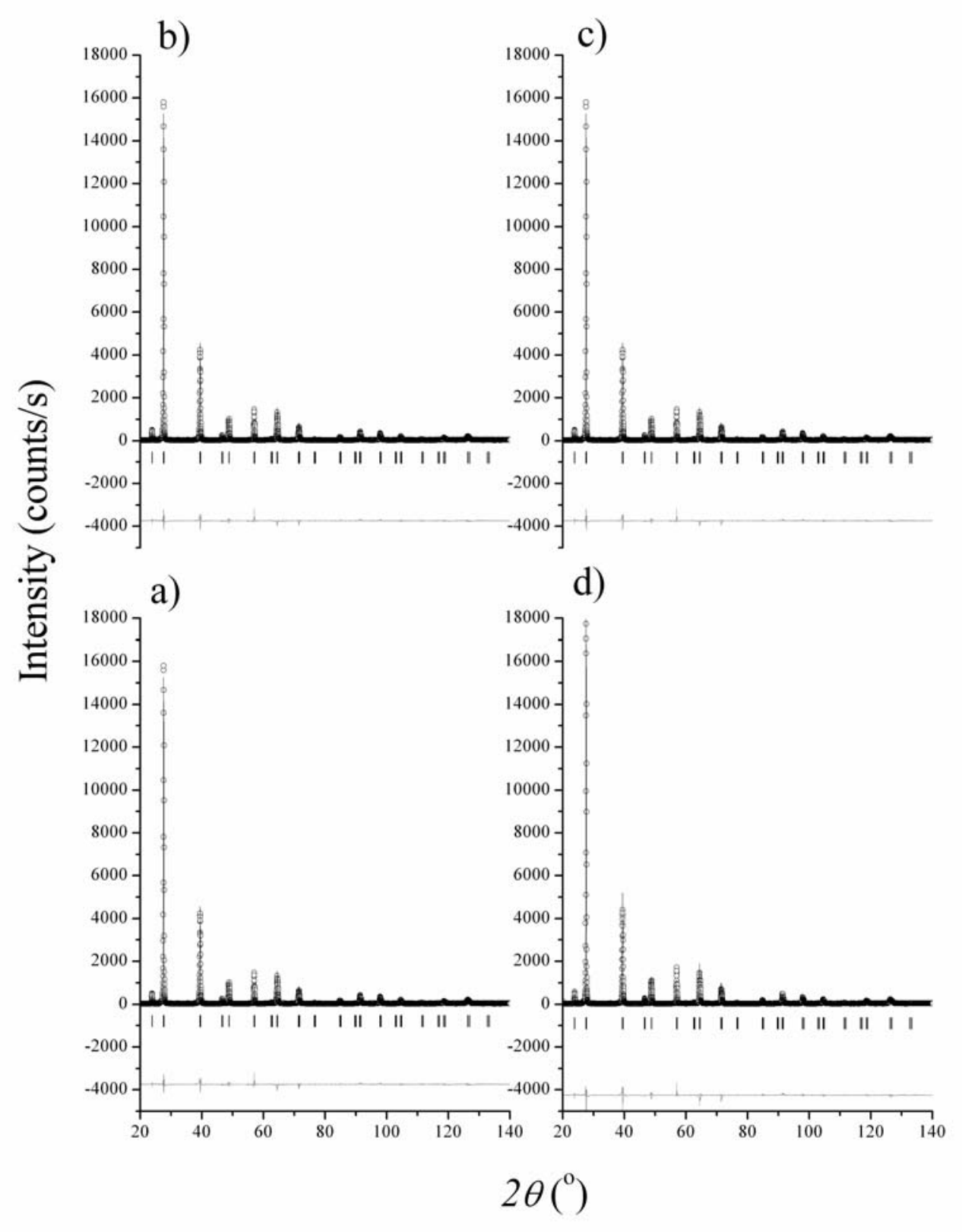

Figure 1. The observed (circles), calculated (continuous line) and difference powder diffraction profiles for: a) undoped $\mathrm{PbTe}, \mathrm{b}) \mathrm{PbTe}$ doped with $\mathrm{B} 1 \mathrm{at} \%$, c) PbTe doped with B 3 at $\%$ and d) PbTe doped with B 8 at $\%$. 
$\mathrm{Pb}$ atoms have been observed to be larger than those of Te atoms, which can indicate thermal vibration increment of the $\mathrm{Pb}$ sublattice to that of the Te sublattice, especially in samples doped with 1 and $8 \%$. However, this is not an unusual phenomenon. Even in pure $\mathrm{PbTe}$ crystals, the relatively large anharmonic thermal vibrations of $\mathrm{Pb}$ lattice to that of the Te sublattice along the $[h h h]$ direction were observed [3]. According to the same data these thermal vibrations of $\mathrm{Pb}$ atoms are enhanced by the alloying elements Ge or Sn and make a lattice unstable. Unit-cell and isotropic displacement parameters in comparison to the literature data [4] are given in Table 3.

Table 3. Rietveld refinement unit-cell and isotropic displacement parameters in comparison to the literature data.

\begin{tabular}{llllcc}
\hline & PbTe & $\begin{array}{c}P b T e+B \\
(1 \%)\end{array}$ & $\begin{array}{c}\text { PbTe+B } \\
(3 \%)\end{array}$ & $\begin{array}{c}\text { PbTe+B } \\
(8 \%)\end{array}$ & $\begin{array}{c}\text { YASUTOSHI } \\
\text { et al. }(1987)\end{array}$ \\
\hline $\mathrm{B}_{\text {iso }} \mathrm{Pb}\left(\AA^{2}\right)$ & $2.037(2)$ & $2.661(1)$ & $2.109(7)$ & $2.648(1)$ & $1.61(2)$ \\
$\mathrm{B}_{\text {iso }} \mathrm{Te}\left(\AA^{2}\right)$ & $1.126(2)$ & $1.319(2)$ & $1.267(1)$ & $1.426(6)$ & $1.11(1)$ \\
$\mathrm{B}$ iso $\mathrm{Pb} / \mathrm{B}_{\text {iso }} \mathrm{Te}$ & 1.809 & 2.017 & 1.665 & 1.856 & 1.45 \\
$a(\AA)$ & $6.4603(2)$ & $6.4605(2)$ & $6.4601(7)$ & $6.4605(2)$ & $6.4541(9)$ \\
$V\left(\AA^{3}\right)$ & $269.62(1)$ & $269.65(2)$ & $269.60(5)$ & $269.65(1)$ & 268.85 \\
\hline
\end{tabular}

\section{Microstructural measurements}

Microstructural parameter calculations were measured in crystallographic directions as follows: [h00]-(200), (400), (600); [hh0]-(220) (440); [hhh]-(111), (222), (333) (444); [hkk]-(311), (622) [h00] and [hh0] layers consist of both $\mathrm{Pb}$ and Te atoms while $[h h h]$ and $[h k k]$ layers consist of either $\mathrm{Pb}$ or Te atoms. So, the (311) layer consists entirely of $\mathrm{Te}$ atoms and the (622) one of $\mathrm{Pb}$ atoms. Likewise, (111) and (333) layers consist entirely of Te atoms; the (222) and (444) layers consist entirely of $\mathrm{Pb}$ atoms. Diffraction-line broadening measurements containing both crystallite domain sizes and microstrain parameters obtained by the Warren-Averbach and the simplified integral-breadth methods are given in Tables 4-7. 
Table 4. Crystallite domain sizes obtained by the Warren-Averbach method.

\begin{tabular}{|c|c|c|c|c|c|}
\hline \multicolumn{6}{|c|}{$D(\AA)$} \\
\hline & & $h 00$ & $h h 0$ & $h h h$ & $h k k$ \\
\hline \multirow[t]{2}{*}{$\mathrm{PbTe}$} & $<D>_{S} *$ & $480 \pm 36$ & $357 \pm 29$ & $466 \pm 64$ & $163 \pm 82$ \\
\hline & $<D>_{v}{ }^{* *}$ & $484 \pm 14$ & $443 \pm 19$ & $484 \pm 27$ & $315 \pm 113$ \\
\hline \multirow[t]{2}{*}{$\mathrm{PbTe}+\mathrm{B}(1 \%)$} & $<D>_{s}$ & $498 \pm 43$ & $428 \pm 38$ & $476 \pm 46$ & $312 \pm 25$ \\
\hline & $<D>_{v}$ & $543 \pm 20$ & $482 \pm 20$ & $546 \pm 24$ & $421 \pm 20$ \\
\hline \multirow[t]{2}{*}{$\mathrm{PbTe}+\mathrm{B}(3 \%)$} & $<D>_{s}$ & $417 \pm 46$ & $339 \pm 30$ & $394 \pm 63$ & $235 \pm 18$ \\
\hline & $<D>_{v}$ & $406 \pm 17$ & $362 \pm 14$ & $407 \pm 26$ & $314 \pm 14$ \\
\hline \multirow[t]{2}{*}{$\mathrm{PbTe}+\mathrm{B}(8 \%)$} & $<D>_{s}$ & $554 \pm 38$ & $430 \pm 54$ & $448 \pm 51$ & $330 \pm 41$ \\
\hline & $<D>_{v}$ & $583 \pm 16$ & $536 \pm 36$ & $545 \pm 46$ & $479 \pm 38$ \\
\hline
\end{tabular}

* Surface-weighted domain size. ** Volume- weighted domain size.

Table 5. Microstrain parameters obtained by the Warren-Averbach method.

\begin{tabular}{llcccc}
\hline & \multicolumn{5}{c}{$\left\langle\varepsilon^{2}\right\rangle^{1 / 2}\left(10^{-3}\right)$} \\
$\mathrm{PbTe}$ & $\left\langle\varepsilon^{2}\right\rangle_{\left\langle D_{S}\right\rangle / 2}^{1 / 2} *$ & $0.89 \pm 0.03$ & $0.99 \pm 0.05$ & $0.66 \pm 0.05$ & $0.72 \pm 0.02$ \\
& $\left\langle\varepsilon^{2}\right\rangle_{\left\langle D_{V}\right\rangle / 2}^{1 / 2} * *$ & $0.88 \pm 0.02$ & $0.89 \pm 0.04$ & $0.68 \pm 0.05$ & $0.75 \pm 0.02$ \\
$\mathrm{PbTe}+\mathrm{B}(1 \%)$ & $\left\langle\varepsilon^{2}\right\rangle_{\left\langle D_{S}\right\rangle / 2}^{1 / 2}$ & $0.95 \pm 0.03$ & $1.05 \pm 0.03$ & $0.77 \pm 0.03$ & $0.84 \pm 0.05$ \\
& $\left\langle\varepsilon^{2}\right\rangle_{\left\langle D_{V}\right\rangle / 2}^{1 / 2}$ & $0.91 \pm 0.02$ & $0.94 \pm 0.03$ & $0.72 \pm 0.03$ & $0.73 \pm 0.04$ \\
& $\left\langle\varepsilon^{2}\right\rangle_{\left\langle D_{S}\right\rangle / 2}^{1 / 2}$ & $1.18 \pm 0.04$ & $1.33 \pm 0.04$ & $0.91 \pm 0.06$ & $0.99 \pm 0.07$ \\
& $\left\langle\varepsilon^{2}\right\rangle_{\left\langle D_{V}\right\rangle / 2}^{1 / 2}$ & $1.19 \pm 0.04$ & $1.29 \pm 0.04$ & $0.89 \pm 0.06$ & $0.86 \pm 0.06$ \\
$\mathrm{PbTe}+\mathrm{B}(3 \%+\mathrm{B}(8 \%)$ & $\left\langle\varepsilon^{2}\right\rangle_{\left\langle D_{S}\right\rangle / 2}^{1 / 2}$ & $0.80 \pm 0.02$ & $0.89 \pm 0.06$ & $0.64 \pm 0.01$ & $0.68 \pm 0.08$ \\
& $\left\langle\varepsilon^{2}\right\rangle_{\left\langle D_{V}\right\rangle / 2}^{1 / 2}$ & $0.78 \pm 0.02$ & $0.80 \pm 0.05$ & $0.53 \pm 0.01$ & $0.56 \pm 0.07$ \\
\hline
\end{tabular}

* Root-mean-square microstrain (RMSM) averaged over the distance $\left\langle D>_{s}\right.$.

** RMSM averaged over the distance $\left\langle D>_{\nu}\right.$. 
Table 6. Crystallite domain sizes obtained by the simplified integral-breadth methods.

\begin{tabular}{|c|c|c|c|c|c|}
\hline & & $D(\AA)$ & & & \\
\hline & & $h 00$ & $h h 0$ & $h h h$ & $h k k$ \\
\hline \multirow[t]{3}{*}{$\mathrm{PbTe}$} & $<D_{C-C}>^{*}$ & 524 & 491 & 467 & 271 \\
\hline & $<D_{C-G}>* *$ & 454 & 446 & 446 & 323 \\
\hline & $<D_{G-G}>* * *$ & 451 & 443 & 443 & 319 \\
\hline \multirow[t]{3}{*}{$\mathrm{PbTe}+\mathrm{B}(1 \%)$} & $<D_{C-C}>$ & 652 & 619 & 569 & 454 \\
\hline & $<D_{C-G}>$ & 523 & 509 & 463 & 421 \\
\hline & $<D_{G-G}>$ & 506 & 493 & 460 & 420 \\
\hline \multirow[t]{3}{*}{$\mathrm{PbTe}+\mathrm{B}(3 \%)$} & $<D_{C-C}>$ & 469 & 441 & 403 & 317 \\
\hline & $<D_{C-G}>$ & 384 & 374 & 366 & 311 \\
\hline & $<D_{G-G}>$ & 375 & 368 & 361 & 311 \\
\hline \multirow[t]{3}{*}{$\mathrm{PbTe}+\mathrm{B}(8 \%)$} & $<D_{C-C}>$ & 672 & 638 & 617 & 502 \\
\hline & $<D_{C-G}>$ & 562 & 551 & 539 & 479 \\
\hline & $<D_{G-G}>$ & 555 & 544 & 538 & 478 \\
\hline
\end{tabular}

Volume-averaged domain size from the simplified integral-breadth methods: * Cauchy-Cauchy function, ** Cauchy-Gauss function, *** Gauss-Gauss function.

Microstrain parameters of both the Warren-Averbach and the simplified integralbreadth methods increase up to the sample doped with $3 \%$, to be followed by a rapid decrease in the sample doped with $8 \%$ of $\mathrm{B}$. This trend is noticeable along each measured crystallographic direction. The referred phenomenon can be explained by B atoms, sited in PbTe structure, in two different manners. Besides, microstrain parameters in the directions, consisting of layers with homogeneous atoms, are much lower in comparison to the parameters with heterogeneous atoms. Taking into consideration all these facts, the basic assumption is that high microstrain values appear as the result of vacancy arrangements in the directions where the layers consist of heterogeneous atoms $(\mathrm{Pb}$ and $\mathrm{Te})$. The point to be emphasised is that the values of the kind were measured in all samples, meaning they are not resulting from B atoms disposition. If so, such values 
would not be measured in the sample doped with $8 \%$.

Table 7. Microstrain parameters obtained by the simplified integral-breadth methods.

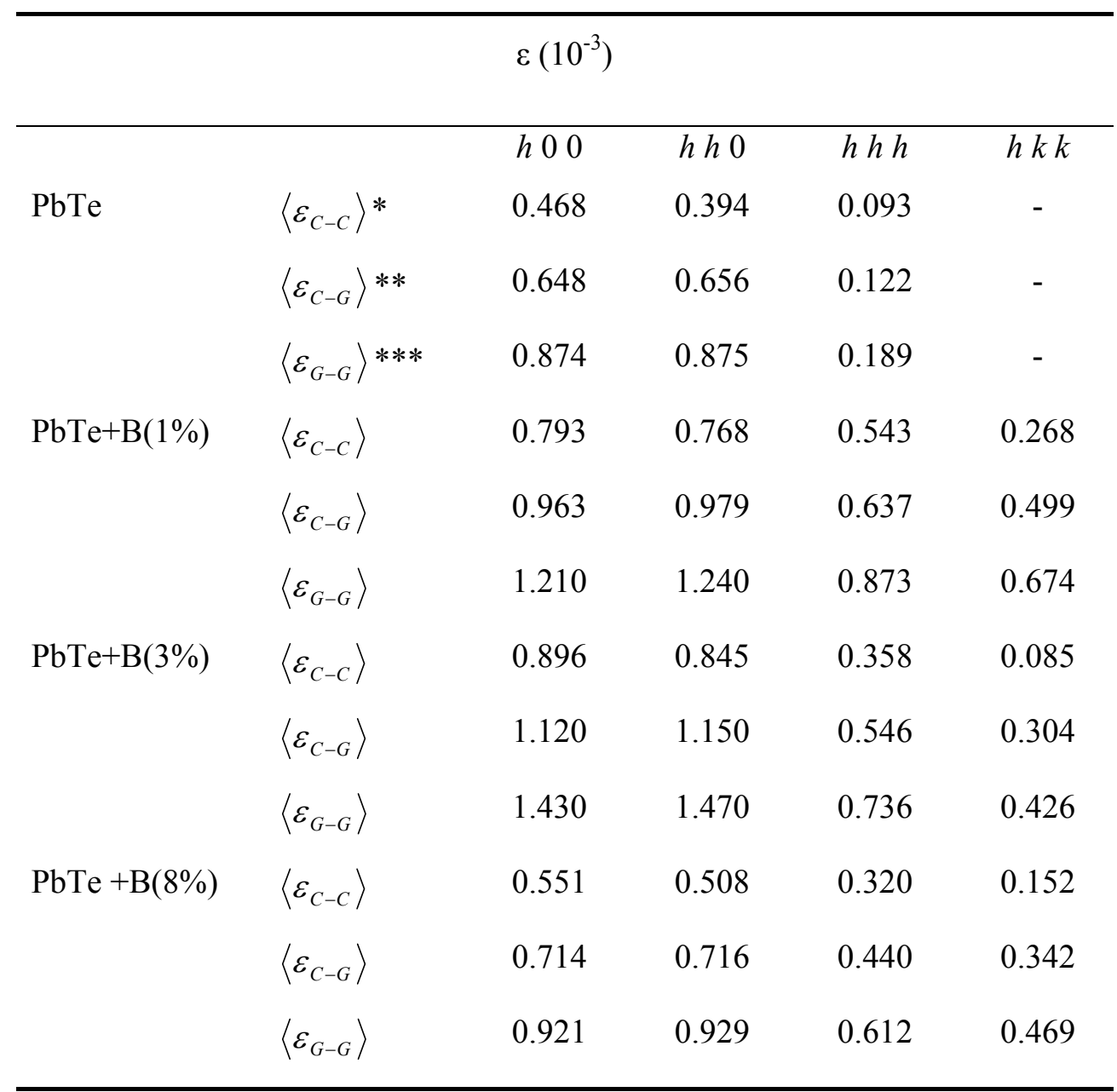

Upper limit of microstrain from the simplified integral-breadth methods: * CauchyCauchy function, ** Cauchy-Gauss function, *** Gauss-Gauss function.

Generally, crystallite domain sizes both for the Warren-Averbach and the simplified integral-breadth methods show higher crystallinity degree in the samples doped with 1 and $8 \%$ (these two samples have also the highest isotropic displacement parameters) and lower one in a pure sample and the sample doped with $3 \%$. Therefore, the crystallinity degree increases up to the sample doped with $1 \%$ of B; aftermath it decreases in the sample doped with $3 \%$ of $\mathrm{B}$, and increases again in the sample doped with $8 \%$ of $\mathrm{B}$. Likewise, this trend may be noticed in microstrain parameter calculations, along each measured crystallographic direction. Graphic 
illustrations of diffraction-line broadening measurements are shown in Figures 2 and 3.

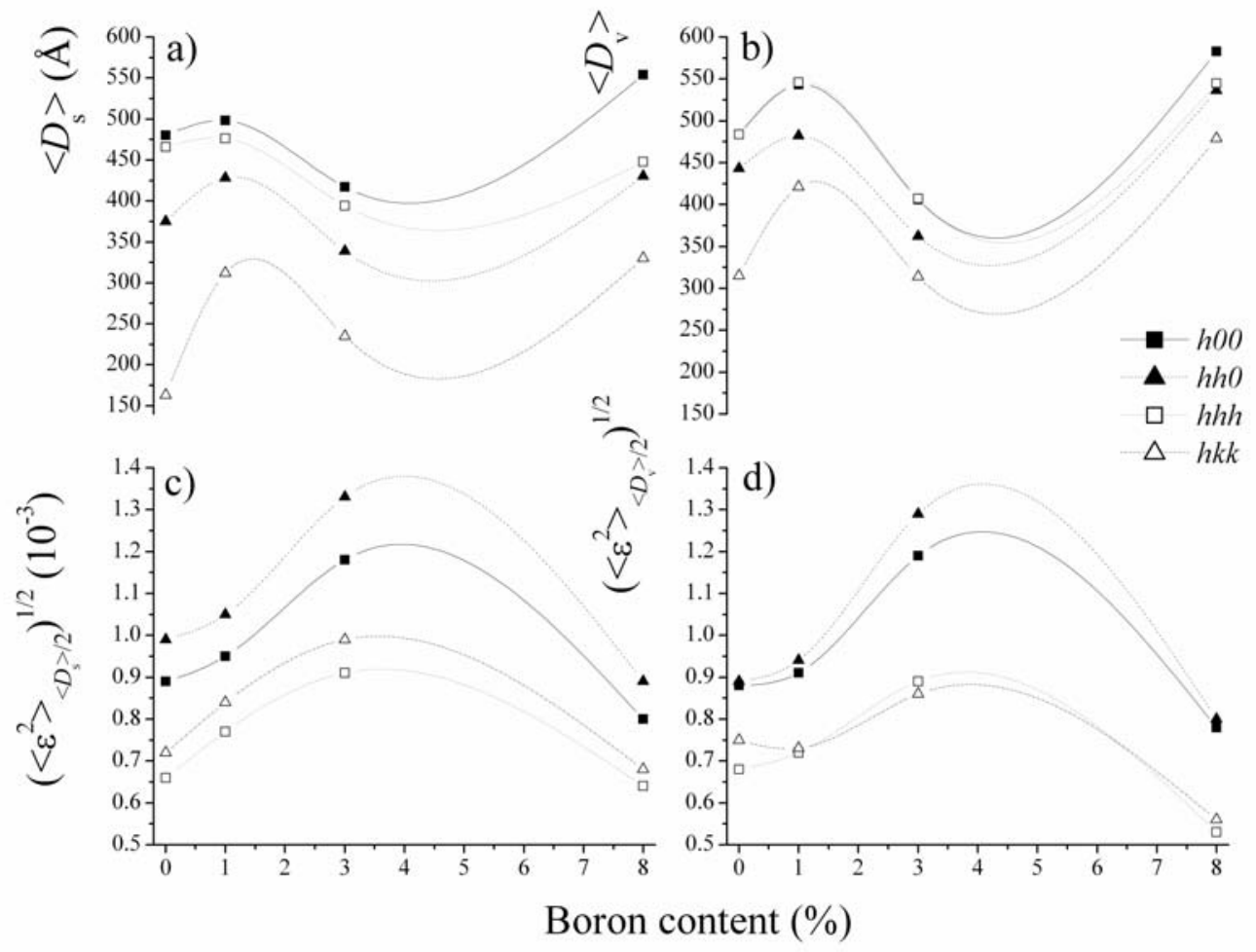

Figure 2. Graphic illustrations of crystallite: a) surface-weighted domain sizes, b) volume-weighted domain sizes, and c) RMSM averaged over the distance $<D>_{s}$, d) RMSM averaged over the distance $\left\langle D>_{v}\right.$ obtained by WarrenAverbach method.

\section{CONCLUSION}

The achieved results of structural and microstructural measurements, par exellence unit-cell and microstrain parameters indicate that $\mathrm{B}$ atoms embedding did not generate disorder in PbTe-type structure. The disorder increases until the sample has been doped with $3 \%$ and then rapidly falls down with the sample doped with $8 \%$ of B. So, if contents of B atoms in the PbTe structure were limited they would be expelled as an 


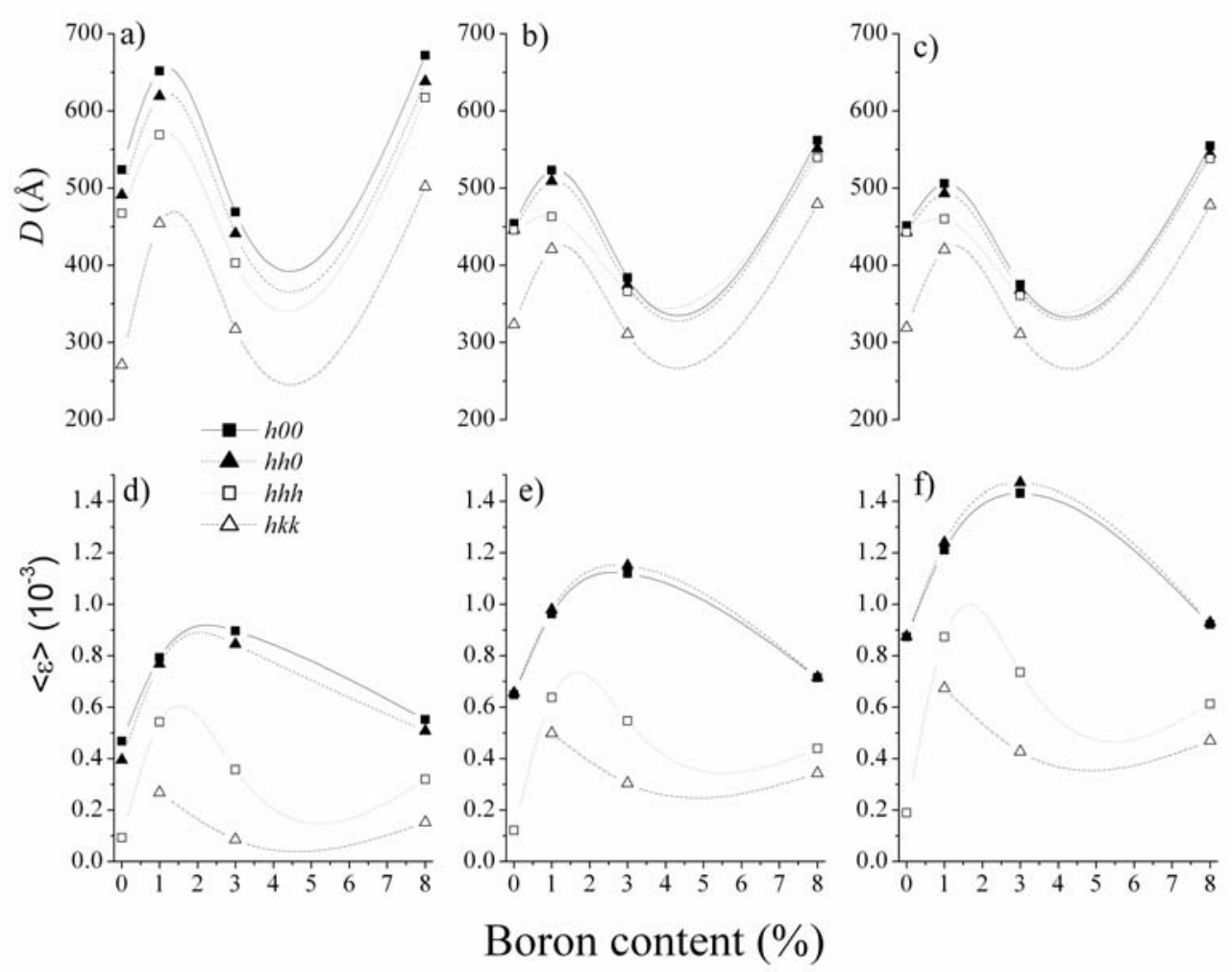

Figure 3. Graphic illustrations of volume-averaged domain sizes: a) Cauchy-Cauchy function, b) Cauchy-Gauss function, c) Gauss-Gauss function, and upper limit of microstrains: d) Cauchy-Cauchy function, e) Cauchy-Gauss function, f) Gauss-Gauss function obtained by the simplified integralbreadth methods.

extra phase in the sample doped with $8 \%$. Have B atoms occupied interstitial site-positions or filled-up vacancies or maybe both? Since these are real crystals, the crystal lattice defects are expected to appear. Schottky-type defects appear when atoms or ions are of approximate dimensions; consequently, the atom distribution in interstitial site-positions is difficult one. Frenkel-type defects consist of either interstitial atoms or ions and vacancies in a crystal lattice. Small cations and big anions are favoured for this type of defects. The foremost assumption is that B atoms, mostly already filled-up, formed vacancies in layers with the highest microstrain 
parameters: [h00] and $[h h 0]$. Since $\mathrm{Pb}$ and $\mathrm{Te}$ atoms are of approximate radii, Schottky-type defects are expected. This means B atoms sited unattended vacancies. Considering $\mathrm{B}$ atomic radius, which is in comparison to $\mathrm{Pb}$ and Te atoms very small one, a certain amount of B atoms must have sited interstitially into the structure. Higher values of isotropic displacement parameters of doped samples in comparison to the pure one can be explained in the manner that B atoms increased overall values of $\mathrm{Pb}$ and $\mathrm{Te}$ atoms. So, the crucial conclusion is that $\mathrm{B}$ atoms predominantly filledup vacancies and, to less extent, degree sited into $\mathrm{PbTe}$ structure interstitially.

\section{ACKNOWLEDGMENTS}

The authors are gratefully acknowledged to Professor Stevan Đurić and academician Pantelija Nikolić, who donated the samples and his associates from the Serbian Academy of Sciences and Arts for their thorough comments and suggestions, which significantly improved this paper.

\section{REFERENCES}

[1] BAlZAR, D., LeDBETTER, H., 1997, "Software for comparative analysis of diffraction line boadening." Advances in X - ray analysis, vol. 39, edited by V. Gilfrich et al., Plenum Press, New York, pp. 457 - 464.

[2] RodrigUeZ - CARVAJAL, J., 1995, “User's guide to program FULLPROF.” 2004-LLBJRC (Laboratorie León Brillouin, CEA-CNRS, Centre d'Etudes de Saclay, Gif sur Yvette, France).

[3] Yasutoshi, N., Shigeru, O., Shoishi, S. \& Yoshiniko, S., 1983, "Charge distribution and atomic thermal vibrations in lead chalcogenide crystals." Acta Cryst., B39, pp. $312-317$.

[4] yasutoshi, N., Katashi, M., Shoishi, S., Yoshiniko, S., Koshiro, T \& IwaO, I. 1987, "Temperature dependence of atomic thermal parameters of lead chalcogenides, PbS, PbSe, PbTe.” Acta Cryst., C43, pp. 1443 - 1445. 